\title{
Is Having Hearing Loss Fundamentally Different? Multigroup Structural Equation Modeling of the Effect of Cognitive Functioning on Speech Identification
}

\author{
Erik Marsja ${ }^{1,2}$ Victoria Stenbäck, ${ }^{1,2}$ Shahram Moradi, ${ }^{1,2,3}$ Henrik Danielsson, ${ }^{1,2}$, \\ and Jerker Rönnberg ${ }^{1,2}$
}

\begin{abstract}
Objectives: Previous research suggests that there is a robust relationship between cognitive functioning and speech-in-noise performance for older adults with age-related hearing loss. For normal-hearing adults, on the other hand, the research is not entirely clear. Therefore, the current study aimed to examine the relationship between cognitive functioning, aging, and speech-in-noise, in a group of older normal-hearing persons and older persons with hearing loss who wear hearing aids.
\end{abstract}

Design: We analyzed data from 199 older normal-hearing individuals (mean age $=61.2$ ) and 200 older individuals with hearing loss (mean age $=60.9$ ) using multigroup structural equation modeling. Four cognitively related tasks were used to create a cognitive functioning construct: the reading span task, a visuospatial working memory task, the semantic word-pairs task, and Raven's progressive matrices. Speechin-noise, on the other hand, was measured using Hagerman sentences. The Hagerman sentences were presented via an experimental hearing aid to both normal hearing and hearing-impaired groups. Furthermore, the sentences were presented with one of the two background noise conditions: the Hagerman original speech-shaped noise or four-talker babble. Each noise condition was also presented with three different hearing processing settings: linear processing, fast compression, and noise reduction.

Results: Cognitive functioning was significantly related to speech-innoise identification. Moreover, aging had a significant effect on both speech-in-noise and cognitive functioning. With regression weights constrained to be equal for the two groups, the final model had the best fit to the data. Importantly, the results showed that the relationship between cognitive functioning and speech-in-noise was not different for the two groups. Furthermore, the same pattern was evident for aging: the effects of aging on cognitive functioning and aging on speech-in-noise were not different between groups.

Conclusion: Our findings revealed similar cognitive functioning and aging effects on speech-in-noise performance in older normal-hearing and aided hearing-impaired listeners. In conclusion, the findings support the Ease of Language Understanding model as cognitive processes play a critical role in speech-in-noise independent from the hearing status of elderly individuals.

Key words: Aging, Cognitive functioning, Raven's, Speech in noise, Structural equation modeling, Working memory.

${ }^{1}$ Division of Disability Research, Department of Behavioral Sciences and Learning, Linköping University, Linköping, Sweden; ${ }^{2}$ Linnaeus CENTRE HEAD, The Swedish Institute for Disability research, Linköping University, Linköping, Sweden; and ${ }^{3}$ Faculty of Health and Social Sciences, Department of Health, Social and Welfare Studies, University of South-Eastern Norway, Porsgrunn, Norway.

Copyright (c) 2022 The Authors. Ear \& Hearing is published on behalf of the American Auditory Society, by Wolters Kluwer Health, Inc. This is an open access article distributed under the Creative Commons Attribution License 4.0 (CCBY), which permits unrestricted use, distribution, and reproduction in any medium, provided the original work is properly cited.

\begin{abstract}
Abbreviations: $\mathrm{BIC}=$ Bayesian Information Criterion; $\mathrm{CFI}=\mathrm{com}-$ parative fit index; ELU = ease of language use; FIML = full information maximum likelihood; $\mathrm{HI}=$ hearing impaired; $\mathrm{HL}=$ hearing level; $\mathrm{LRT}=$ likelihood ratio test; $\mathrm{NH}=$ normal hearing; $\mathrm{PTA}=$ pure tone average; RAMBPHO = Rapid, Automatic, Multimodal Binding of Phonology; RMSEA = root mean square error of approximation; SD = standard deviation; SEM = structural equation modeling; SNR = signal to noise ratio; SPL = sound pressure level; SRMR = standardized root mean residual; $S R T=$ speech reception threshold; $S S N=$ speechshaped noise; TLI = Tucker-Lewis Index; VSWM = visuospatial working memory; WAIS = Weschler Adult Intelligence ScaleL WASI = Wechsler Abbreviated Scale of Intelligence; $\mathrm{WM}=$ working memory; $\mathrm{WMC}=$ Working Memory Capacity.
\end{abstract}

(Ear \& Hearing XXX;XX;00-00)

\section{INTRODUCTION}

Aging is associated with a decrease in sensory, cognitive, and motor functions that affects daily functioning of older individuals. One such function affected by aging is the perception of speech signals, particularly when the speech signal is degraded by background noise or competing speech.

Aging affects both auditory functions (e.g., increased hearing thresholds; Gates \& Cooper 1991; Wiley et al. 2008; poorer auditory temporal processing; Fitzgibbons \& Gordon-Salant 2001; Pichora-Fuller et al. 2006) and cognitive functions (e.g., lower working memory capacity; Salthouse \& Babcock 1991; Wingfield et al. 1988, reduced attentional capacity; e.g., Graham \& Burke 2011; McAvinue et al. 2012). In two studies by Baltes and Lindenberger (1997) and Lindenberger \& Baltes (1994), aging was shown to have a relatively general effect, rather than a specific effect, on sensory and cognitive functions across the adult life span, particularly in older age. In a more recent study, Danielsson et al. (2019) studied the association between different types of auditory constructs (thresholds, gap detection, and temporal-order identification) and four different types of cognitive tests (semantic long-term memory, episodic long-term memory, cognitive processing speed, and working memory) in 213 normal-hearing listeners. The results showed that age had varied effects on different types of auditory and cognitive tasks. The strongest effects were on cognitive speed and hearing thresholds, with moderate effects on episodic longterm memory, working memory (WM), and temporal-order identification. The weakest effect was on gap detection, with zero effect on semantic long-term memory. Danielsson et al. (2019) also found that the effect of hearing on cognition was weak in normal-hearing listeners.

Previous research has shown that both auditory and cognitive functions are pivotal components in speech intelligibility 
in noise for older individuals (e.g., Besser et al. 2015; Lunner \& Sundwall-Thorén 2007; Moore et al. 2014; Stenbäck et al. 2015; see, however, Schoof \& Rosen 2014 for results showing no auditory decline). In a systematic review and meta-analysis, Dryden et al. (2017) reported a correlation of $r=0.3$ between speech-in-noise identification and general cognitive performance (i.e., WM, attention, memory, etc., pooled together). One of the most studied cognitive domains is WM. Briefly defined, WM is a system with limited capacity in which information is stored temporarily. The stored information is used for executing complex tasks such as reasoning, learning, and comprehension. A person's working memory capacity (WMC) is their ability to store and process information simultaneously (Baddeley 2012).

Ample research data support the relationship between WMC and speech intelligibility in noise in unaided conditions (see Dryden et al. 2017). A majority of the literature found this link in people with hearing loss (e.g., Arehart et al. 2013; Foo et al. 2007; Lunner 2003). In a review by Akeroyd (2008), both hearing loss and WMC were found to be predictors of speechin-noise identification. Moreover, there is also evidence that suggesting that normal-hearing individuals use explicit cognitive processing (e.g., WMC) in challenging listening conditions as. For instance, Michalek et al. (2018) studied young normal-hearing individuals and reported that more challenging signal-tonoise ratios in babble noise increased participants' dependence on WMC. However, in a review of 19 studies, Füllgrabe and Rosen (2016b) found that WMC accounted for less than $2 \%$ of the variance in speech-in-noise performance for young normalhearing listeners. In another study, Füllgrabe and Rosen (2016a) reported that the interaction of auditory and cognitive function in speech-in-noise performance was more evident in older than young normal-hearing individuals.

One theory concerning cognition and speech identification is the ease of language understanding (ELU) model. According to the ELU model (Rönnberg et al. 2013, 2019, 2021), background noises make it difficult to map speech signals with corresponding breakdown in the phonological/lexical representations in semantic memory. This, in turn, is assumed to increase the demand for explicit cognitive resources (e.g., WM and attention) to process degraded speech input for correct identification. In the framework of the ELU, speech input is first processed in the RAMBPHO (rapid, automatic, multimodal binding of phonology) buffer. RAMBPHO binds representations based on multimodal information relating to syllables. If there is a match between the input and phonological and lexical long-term representation, lexical access is successful with less or no demand on explicit cognitive resources. On the other hand, if there is a mismatch between the input and the long-term representations (e.g., due to background noise or hearing loss) the RAMBPHO buffer feeds it forward to WM and executive functions to help disambiguate the identity of ambiguous speech signals (Rönnberg et al. 2013, 2019).

In the ELU model, WM has two roles when it comes to language understanding: prediction and postdiction (Rönnberg et al. 2013, 2019). The latter role, postdiction, is the cognitive mechanism used when a phonological mismatch has occurred. Here, WM is used for inference-making concerning the identity of speech input. The predictive role of WM concerns priming and pre-tuning the RAMBPHO buffer and focusing of attention. Finally, postdiction and prediction are thought to be as important for individuals with or without hearing loss.
Speech-in-noise tests are a valuable tool in the clinic in how people process and recognize speech under adverse listening, conditions which could also be generalized to real-life communication situations. They have been used extensively in research, for example, in research on the rehabilitation of people with hearing loss (Rönnberg et al. 2019). For example, the Swedish Hagerman sentences (Hagerman 1982; Hagerman \& Kinnefors 1995) consist of five words in a closed-set structure. Whereas the grammatical structure of the sentences is predictable, it is not possible to predict the words themselves. Importantly, the words in the Hagerman sentences cannot easily be guessed if they are not heard clearly. The noise in the Hagerman speechin-noise test can be set to target speech reception thresholds (SRTs) of $50 \%$ or $80 \%$ word recognition. Stenbäck et al. (2016) showed that older normal-hearing individuals with high WMC perform better in the Hagerman speech-in-noise test compared with older individuals with lower WMC. However, for younger individuals, Stenbäck et al. (2016) failed to find a significant effect of WMC. These results are further corroborated by a more recent brain imaging study by Giroud et al. (2021). In their study, the authors found that thicker frontal lobe regions were related to higher WMC and better performance in the speechin-noise test for older individuals.

The research is not entirely clear concerning the relationship between WMC and SRTs (e.g., 50\% and 80\% recognition). For example, studies by Larsby et al. (2008) and Lunner and Sundewall-Thorén (2007) suggest that cognitive functioning plays a more critical role at $80 \%$ SRT compared with $50 \%$ SRT in older people with hearing loss. However, in a study of normal-hearing individuals, Gordon-Salant and Cole (2016) found a relationship between WMC and speech-in-noise identification at $50 \%$ recognition, using a word recognition task. For individuals with hearing impairment, it has further been shown that aided speech recognition in noise has a relationship with WMC (e.g., Foo et al. 2007). What seems to be important for speechin-noise identification is whether the WM tests, in general, are dual tasks. That is, they are measuring processes similar to language comprehension, that is, involving both storage and processing of information (see Rönnberg et al. 2016).

Furthermore, a large body of research has shown a substantial relationship between WMC and general fluid intelligence (e.g., Ackerman et al. 2002; Conway et al. 2002; Engle et al. 1999; Kyllonen \& Christal 1990; Unsworth \& Engle 2005). Fluid intelligence is generally referred to as the ability to solve novel problems using abstract reasoning. Moreover, it is linked to the ability to recognize patterns and engage with the world. Fluid intelligence is often measured using Raven's progressive matrices (Raven 2000). Raven's progressive matrices have been shown to have a relationship with a broad range of WM tests. For example, WM tests such as reading and counting span have been shown to correlate with Raven's progressive matrices (Conway et al. 2002; Engle et al. 1999). Moreover, it has been reported that visuospatial WM and fluid intelligence are related (Kane et al. 2004). A few studies have examined the link between fluid intelligence (mainly using the Wechsler Adult Intelligence ScaleRevised [WAIS-R] or some of its subtests) and speech intelligibility in noise. In a study by Jerger et al. (1989), Digit Symbol test of WAIS-R was correlated with speech in noise identification. However, Humes et al. (1994) reported that factors derived from WAIS-R accounted for no variance to a small portion of the variance for speech-recognition performance. Furthermore, Meister 
et al. (2013) found that a reasoning task related to fluid intelligence was related to a two-talker situation in a dichotic listening task. Moore et al. (2014) analyzed data from the UK BioBank. They found that lower fluid intelligence, as measured by a logical reasoning task, was associated with poorer performance in a speech-in-noise test. Moreover, Füllgrabe et al. (2015) reported significant correlations between the Block Design test (measuring mental speed) from the Wechsler Abbreviated Intelligence Scale (WASI) and speech in noise identification. However, the authors reported a non-significant correlation between Matrix Reasoning (measuring fluid intelligence) and speech in noise identification. In a more recent study by Pronk et al. (2019), poorer performance on Raven's progressive matrices (as a measure of fluid intelligence) was found related to poorer speechin-noise performance. To summarize, fluid intelligence seems likely to be related to speech-in-noise performance as it is generally found to correlate with WMC, a rather established predictor of speech-in-noise identification, and a few studies suggest that fluid intelligence is as well.

Hearing aids cannot fully restore difficulties in language understanding of people with hearing loss (see Dimitrijevic et al. 2004; Moradi et al. 2014). In addition, studies have shown that hearing loss is associated with decline in semantic longterm memory (e.g., Rönnberg et al. 2011), lower cognitive function, and accelerated cognitive decline (e.g., Croll et al. 2021). Further, hearing loss had an adverse effect on the neural brain areas involved in the processing of speech signals (e.g., Husain et al. 2011; Peelle et al. 2011). Taken together, it has been suggested that people with hearing loss, even when using hearing aids, dedicate greater cognitive resources in the process of inference-making to map degraded speech input onto established phonological/lexical representation in semantic longterm memory (see Cardin 2016).

One interesting research question is whether underlying factors that affect speech-in-noise are the same or different in people with normal hearing and hearing loss (in aided listening conditions) when SNRs for presenting speech stimuli are individually adapted (e.g., 50\% and 80\% SRTs). Using multi-group structural equation modeling (SEM), the present study investigated whether the link between cognitive functioning (WM and fluid intelligence) and speech-in-noise identification is similar for individuals with normal hearing and hearing loss. SEM is used to analyze the structural relationship between measured and latent variables. Moreover, SEM and the multi-group variant, in this case, have several advantages compared with simpler statistical methods (e.g., correlation and regression). First, it enables us to set up the latent variable cognitive functioning by using several cognitive tasks. Second, multi-group SEM enables us to test a theoretical model between two groups by estimating models where all parameters are allowed to differ between the groups.

For the present study, we employed data from the n200 study (see Rönnberg et al. 2016 for n200 data in people with hearing loss) to address this question. In short, the n200 study is an ongoing longitudinal study evaluating the interaction of aging, speech intelligibility in noise, and cognition in people with hearing loss, using a larger sample than the majority of previous studies. We analyzed data from two different samples of the n200 study: a group of aided hearing-impaired individuals [or n200-hearing impaired, described in Rönnberg et al. (2016)] and a group of people with normal hearing (or n200-NH) for whom the first data collection has been completed. A broad variety of cognitive, speech, and physiological data were collected in phase one of the project for both hearing-impaired and normal-hearing individuals.

In the present study, given that the Hagerman speech-innoise test is presented to older people with hearing loss in aided listening, we hypothesized similar additive effects of cognitive functioning and aging on speech-in-noise in normal hearing and aided hearing-impaired listeners.

\section{MATERIALS AND METHODS}

\section{Participants}

In the n200 study, 215 normal-hearing adults (all thresholds at and below $3000 \mathrm{~Hz}<20 \mathrm{~dB}$ hearing level in the best ear and $<30 \mathrm{~dB}$ hearing level in the worst ear) and 213 adults with hearing loss participated. See Figure 2 for audiograms for better and worse ears. They all had normal or corrected to normal vision and no known cognitive deficits as screened with the Mini-Mental State Examination (Folstein et al. 1975). In the current study, we only included participants that had completed all three different sessions of the data collection. This means that we analyzed data from 199 normal-hearing adults (97 male) between 42 and 78 years $(M=61.2, S D=8.16)$ and 200 adults (115 male and one unknown) with hearing loss between 33 and 80 years $(M=60.9, S D=8.41)$. The current study was conducted in accordance with the Declaration of Helsinki and was approved by the ethical review board at Linköping University (DNR: 55-09 T122-09).

\section{Measurements}

The Reading Span Test - The participants were presented with The Reading Span Test (e.g., Rönnberg et al. 1989). The test includes a series of short (three words) Swedish sentences presented on a computer screen one word at a time. The participants were instructed to remember the presented sentences and, after each sentence, judge whether the sentence was regular (e.g., The ball bounced far) or nonsense (e.g., The fox wrote poetry). The sentences were presented in sets of two to five sentences, with increasing difficulty after each set. When a set had been presented, the participants were asked to recall either the first or the last word in each sentence, in the correct serial order. In the present study, the total score of recalled items is used as a measure of WMC, irrespective of recall order. Finally, the maximum score on the reading span test was 28 .

Visuospatial Working Memory. - Visuospatial working memory assesses the ability to recall non-verbal working memory information (Rönnberg et al. 2016). The task was to judge whether ellipsoid shapes in a $5 \times 5$ grid of squares were identical and after a sequence had been presented, the participants had to recall where in the grid the shapes were located by drawing them on an empty grid sheet $(5 \times 5)$. The maximum score in the visuospatial working memory test was 42 .

Semantic Word-Pairs Test • Semantic word-pairs (Rönnberg et al. 2016) test evaluates working memory capacity that does not involve syntactic elements in the processing and storage components. The participants were presented with word-pairs visually on a computer screen and asked to decide which of the two words represented a living object. After a set of word-pairs, the participant was asked to recall either the first or the second word of the presented word-pairs. In the Semantic word-pairs test, the maximum score was 42 . 


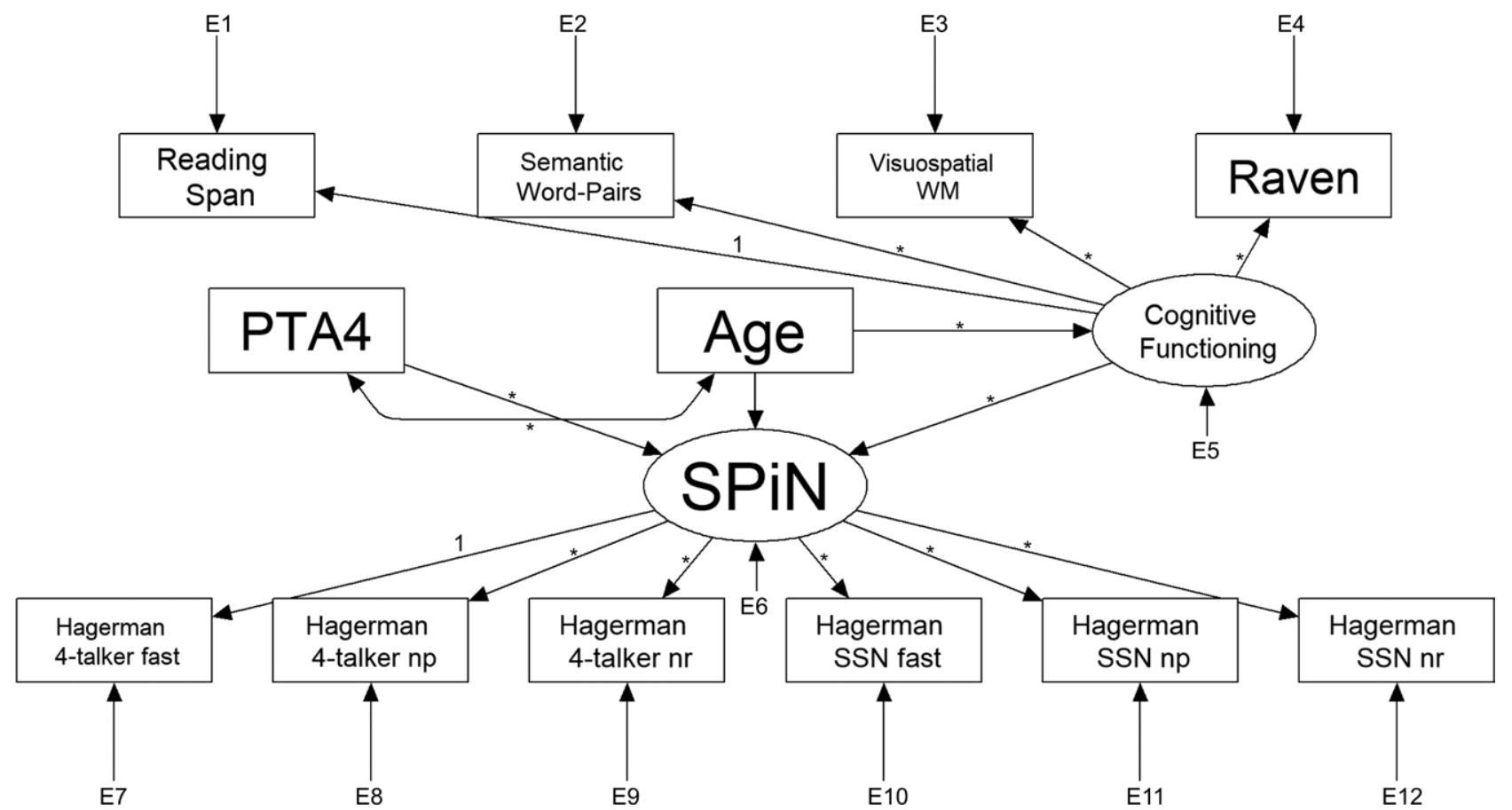

Fig. 1. Theoretical model that was tested. The numbers on the arrows indicate that these relationships are fixed. The asterisks on the arrows indicate that these relationships are freely estimated parameters.

Raven's Advanced Progressive Matrices Test • The current study used three (A, D, and E) out of five sets of Raven's progressive matrices test. The first set (A) was used for practice, and sets $\mathrm{D}$ and $\mathrm{E}$, containing 12 items each, were administered without feedback and with a time limit. The test was scored by the sum of points on sets $\mathrm{D}$ and $\mathrm{E}$ with a 12 as the max score for each set, and the max score for the entire test was 24 .

Hagerman Speech in Noise - The speech material in the Hagerman speech-in-noise test (Hagerman 1982) has a syntactically fixed structure, and all sentences comprise five words. Each word represents a distinct category in the following order: proper noun, verb, number, adjective, and plural noun. Examples of Hagerman sentences are (translated from Swedish) "Peter bought seven light bowls" or "Elsa gave six new gloves". Lists were created by Hagerman (1982) by using commonly used Swedish words (Allén 1970) and names to create 10 sentences with the abovementioned structure. The same words and names occur in various combinations in the lists. In the n200 study (Rönnberg et al. 2016), the Hagerman sentences were presented in a soundattenuated booth (model CA Tegnér) at $65 \mathrm{~dB}$ sound pressure level targeting SRTs for either $50 \%$ or $80 \%$ word recognition using an interleaved method (Brand 2000). The sentences were presented binaurally via inserted earphones with one of two background noise conditions: the Hagerman original speech-shaped noise (SSN) or four-talker babble. The SSN is only slightly modulated (approximately 10\%), and the four-talker babble consists of four different talkers (two men and two women) reading aloud simultaneously from a Swedish newspaper. After each sentence, there was a pause long enough for the participant to repeat the recognized words back verbally. The presentation level of the noise began at $65 \mathrm{~dB}$ signal to noise ratio (SNR), with the SNR increased or decreased by $1 \mathrm{~dB}$ depending on the number of correctly recognized words. Specifically, two correctly identified words in a Hagerman sentence did not increase or decrease in dB SNR. One or zero correctly recognized words resulted in +1 - and $+2-\mathrm{dB}$ SNR, respectively. Three, four, and five correctly identified words resulted in -1, -2-, and -3-dB SNR, respectively. The participants' pure tone audiogram was entered into an experimental hearing aid prior to testing [see. Rönnberg et al. (2016) supplemental material for further details] to present the speech in noise test in various hearing processing settings. In order to have similar experimental settings in participants with normal hearing and participants with hearing loss, we used the experimental hearing aid as well for people with normal hearing (instead of using loudspeakers or earphones). Three different hearing processing settings were used: (1) linear processing (hereafter referred to as $\mathrm{np}$ ), (2) fast compression (referred to as fast), and (3) noise reduction (referred to as $\mathrm{nr}$ ). Each participant was given two lists (10 sentences in each list) as a practice round. According to Hagerman and Kinnefors (1995), one training list before each test session reduces any training effects. In the current study, only $50 \%$ speech recognition was used as a measure of speech recognition in noise. To obtain SNRs for each of the conditions in the Hagerman test, the average SNRs across three lists were calculated.

\section{Statistical Analysis}

This paper was prepared using RMarkdown and Papaja (Aust $\&$ Barth 2020). All data were processed and analyzed within the R statistical programming environment ( $\mathrm{R}$ Core Team 2021). Structural equation modeling (SEM) was carried out using the r-package lavaan (Rosseel 2012) and visualized using semPlot (Epskamp 2019).

The amount of missing data was low (Raven's progressive matrices as the worst case with $2.5 \%$ missing in the normal hearing group) and were therefore treated as missing at random. We used full information maximum likelihood to handle missing data. Full information maximum likelihood missing data handling utilizes all of the available observations for each case 
to compute the likelihood function and has been shown to perform well (Enders \& Bandalos 2001). This method subsequently provides unbiased estimates with minimal standard errors when data are missing at random (Schafer \& Graham 2002).

Conventional fit indices were used to evaluate the model fit in the SEM models. More specifically, we used the comparative fit index, the Tucker-Lewis Index, the standardized root mean residual, Bayesian Information Criterion (BIC), and the root mean square error of approximation (RMSEA). Traditional cutoff criteria with comparative fit index and Tucker-Lewis Index values over 0.95 (Hu \& Bentler 1999) and standardized root mean residual and root mean square error of approximation values under 0.06 to 0.08 were used to indicate acceptable fit (Steiger 2007). BIC was mainly used for comparing different models. This measure is commonly used for selecting among alternative models, and the model with the lowest value is preferred (e.g., Lin et al. 2017; Vrieze 2012). Thus, when selecting the most optimal model, we were more interested in the BIC values along with the measures of fit (e.g., RMSEA).

Chi-square $\left(\chi^{2}\right)$ together with the associated degrees of freedom and $p$-value was reported but not used as a goodness-of-fit criterion because it tends to reject models based on large sample sizes (e.g., MacCallum et al. 1996). The model was evaluated according to suggested optimization using modification indices. We only implemented theoretically sound modifications. Moreover, the different models were also compared using likelihood ratio test (Satorra \& Bentler 2001). Here, it is worth noting that when improving the models, using modification indices, we want the addition to the new model to be significantly better than the old model. However, the likelihood ratio test should not be significant when constraining the model and testing for measurement invariance (e.g., Putnick \& Bornstein 2016). Moreover, since this article focuses on the regression paths and whether they are different between the two groups, we tested for invariance between three models: (1) constraining the factor loadings, (2) constraining the regression weights, and (3) constraining both the factor loadings and the regression weights. Figure 1 depicts the theoretical structural model to be tested in this article.

\section{RESULTS}

\section{Descriptive Statistics and Correlation Matrices}

Tables 1 and 2 show the means, standard deviations, and correlation estimates (i.e., Pearson's product-moment coefficients) for the Hagerman 50\% and cognitive functioning measurements in the normal-hearing and hearing loss groups, respectively. The measured variables that are linked to the latent variable Speechin-Noise (SPiN) in the proposed model all show significant internal correlations. In general, all the SPiN variables show high correlations. Moreover, the correlations between the different cognitive variables are relatively high, and, therefore, we continue with structural equation modeling.

\section{SEM: 50\% Hagerman}

Our final model (see Figure 3), in which we constrained the regression weights to be equal across groups, fit the data well, $\chi^{2}(83)=115.96, p<0.001, R M S E A=0.04(0.02-0.06)$, $C F I=0.98$, and $T L I=0.98$. The final model differed from the others only in constraining the factor loadings and weights. In the first step of optimizing the model, we let the error terms of the observed variables reading span and semantic-word pairs covary. This was followed by also letting the error terms of two Hagerman conditions covary (Hagerman 4-talker fast and SSN fast). Optimization of the models was done by looking at the modification indices, and we only chose the indices that made theoretical sense (and statistically improved the model). Briefly, modification indices, in this case, mean that there is some variance unexplained by observed variables. This variance, in turn, is shared among the variables (e.g., reading span and semanticword pair). All models and their fit measures can be found in Table 3. Furthermore, this table shows that the baseline model shows a poor fit to the data, whereas the model with equal factor loadings and regression weights between the groups shows a good fit. The results from the LRTs confirmed the visual inspection of fit indices. That is, our optimization of the baseline model resulted in significantly better models (i.e., models 2 - 3). Finally, the results also showed that our equality constraints did not change the model (i.e., models 3-6). Likelihood ratios, $p$-values, and squared degrees of freedom can be found in Table 4.

Cognitive functioning was related negatively to SNR, in both groups $(\beta=-0.32$, and $\beta=-0.32$, in normal hearing and hearing aid group, respectively), age was related to both Cognitive functioning, in both groups (negatively related; $\beta=-0.5$ and $\beta=-0.55$, in normal hearing and hearing aid group, respectively) and SNR (positively related; $\beta=0.15$ and $\beta=0.17$ in normal hearing and hearing aid group, respectively). Finally, PTA4 was positively related to $\operatorname{SNR}(\beta=0.42$ and $\beta=0.28$, in

TABLE 1. Mean, standard deviation (within parentheses), and correlation estimates for all variables included in the current study

\begin{tabular}{|c|c|c|c|c|c|c|c|c|c|c|c|c|}
\hline Varialbes & Mean (SD) & 2 & 3 & 4 & 5 & 6 & 7 & 8 & 9 & 10 & 11 & 12 \\
\hline 2 Hagerman nr SSN & $-12.66(1.3)$ & - & 0.46 & 0.63 & 0.65 & 0.53 & -0.1 & -0.13 & -0.06 & -0.22 & 0.35 & 0.28 \\
\hline 4 Hagerman np 4-Talker & $-2.85(1.61)$ & & & - & 0.66 & 0.57 & -0.15 & -0.22 & -0.28 & -0.34 & 0.33 & 0.43 \\
\hline 5 Hagerman nr 4-Talker & $-9.4(1.38)$ & & & & - & 0.5 & -0.17 & -0.2 & -0.28 & -0.26 & 0.34 & 0.39 \\
\hline 6 Hagerman fast 4-Talker & $-2.55(1.68)$ & & & & & - & -0.2 & -0.25 & -0.16 & -0.34 & 0.26 & 0.36 \\
\hline 7 Semantic Word-Pair & $19.01(5.88)$ & & & & & & - & 0.51 & 0.24 & 0.27 & -0.03 & -0.26 \\
\hline 10 Raven & $16.39(4.64)$ & & & & & & & & & - & -0.23 & -0.39 \\
\hline 11 PTA4 & 10.06 (6.13) & & & & & & & & & & - & 0.44 \\
\hline 12 Age & $61.22(8.16)$ & & & & & & & & & & & - \\
\hline
\end{tabular}


TABLE 2. Mean, standard deviation (within parentheses), and correlation estimates for all variables included in the current study

\begin{tabular}{|c|c|c|c|c|c|c|c|c|c|c|c|c|}
\hline Variables & Mean (SD) & 2 & 3 & 4 & 5 & 6 & 7 & 8 & 9 & 10 & 11 & 12 \\
\hline 1 Hagerman np SSN & $-6.25(1.6)$ & 0.65 & 0.72 & 0.73 & 0.66 & 0.6 & -0.12 & -0.14 & -0.18 & -0.33 & 0.4 & 0.32 \\
\hline 2 Hagerman nr SSN & $-11.24(1.67)$ & - & 0.63 & 0.59 & 0.64 & 0.5 & -0.14 & -0.22 & -0.18 & -0.3 & 0.25 & 0.28 \\
\hline 3 Hagerman fast SSN & $-5.93(1.63)$ & & - & 0.67 & 0.67 & 0.69 & -0.16 & -0.18 & -0.24 & -0.26 & 0.38 & 0.3 \\
\hline 5 Hagerman $\mathrm{nr}$ 4-Talker & $-7.95(1.58)$ & & & & - & 0.58 & -0.21 & -0.17 & -0.21 & -0.41 & 0.41 & 0.29 \\
\hline 6 Hagerman fast 4-Talker & $-0.31(1.92)$ & & & & & - & -0.19 & -0.15 & -0.2 & -0.25 & 0.45 & 0.32 \\
\hline 7 Semantic Word-Pair & $17.49(5.4)$ & & & & & & - & 0.45 & 0.39 & 0.31 & -0.09 & -0.26 \\
\hline 10 Raven & $15.51(4.67)$ & & & & & & & & & - & -0.1 & -0.27 \\
\hline 11 PTA4 & 37.27 (10.64) & & & & & & & & & & - & 0.14 \\
\hline 12 Age & $60.89(8.41)$ & & & & & & & & & & & - \\
\hline
\end{tabular}

Hearing aid users.

fast, fast compression; $n p$, linear processing; $n r$, noise reduction, WM, working memory.

normal hearing and hearing aid groups, respectively). For standardized regression coefficients and confidence intervals, see Table 5.

To conclude the result section: of particular interest for the current study is the path between cognition and speech-in-noise. We can see that when setting the regression weights equally between the two groups, the model is a good fit for the data. Furthermore, there is a relationship between cognitive functioning and speech in noise performance that is quite similar regardless of having hearing loss or not.

\section{DISCUSSION}

The current study aimed to examine the additive effects of cognitive functioning, and age, on speech in noise, in a group of normal-hearing individuals and individuals with hearing loss. To this aim, we used multi-group structural equation modeling.

In the current study, we found that cognitive functioning had a significant effect on aided speech-in-noise performance whether an individual has hearing loss or not. More specifically, we found that (1) cognitive functioning affected speech-in-noise identification, and (2) that age had a significant effect on both speech-in-noise and cognitive functioning. Importantly, in the final model, the regression weights between the latent variables were set to be equal. This, in turn, proved to improve the model (i.e., increasing the goodness-of-fit indices), meaning that the effects generalize to both groups.

We reason that one explanation for the similar pattern of results between the two groups is due to successful providing of audibility for people with hearing loss as the SRT values for different settings of Hagerman speech-in-noise test are nearly similar between two groups (see Tables 2 and 3). Humes (2002) reported that auditory functioning played the most important role in speech recognition under unaided conditions. However, Humes et al. (2013) revealed that by providing audibility for older adults, cognitive factors contributed greatly to recognizing speech stimuli. We speculate that different pattern of results can be seen between two groups in

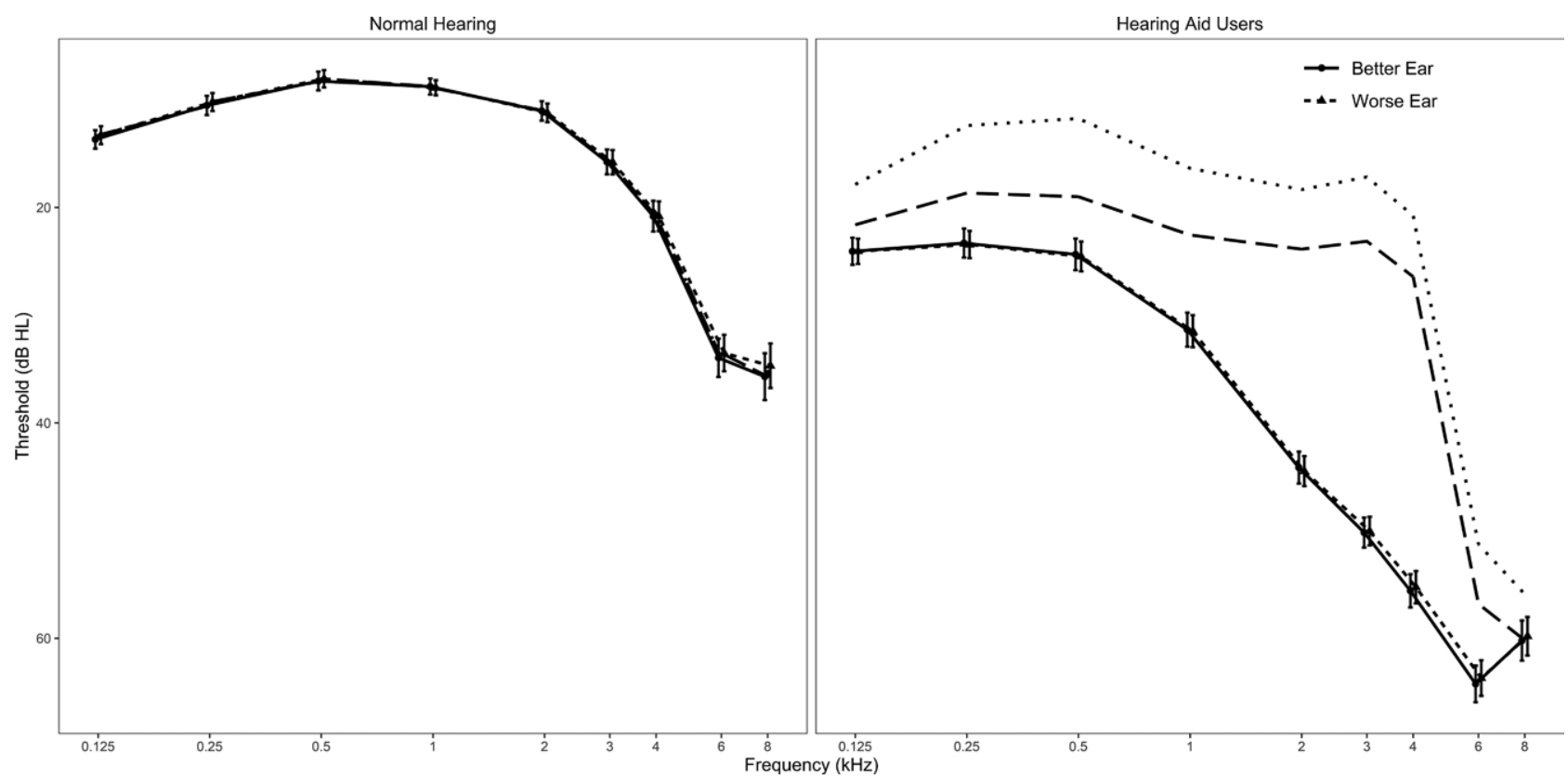

Fig. 2. Audiograms for better and worse ear for the two groups. Error bars are displaying $95 \%$ confidence intervals. The dotted and long dashed lines above the better and worse ear audiograms on the right panel (Hearing Aid Users) depict the gains to the audiogram for linear and fast signal processing, respectively. 

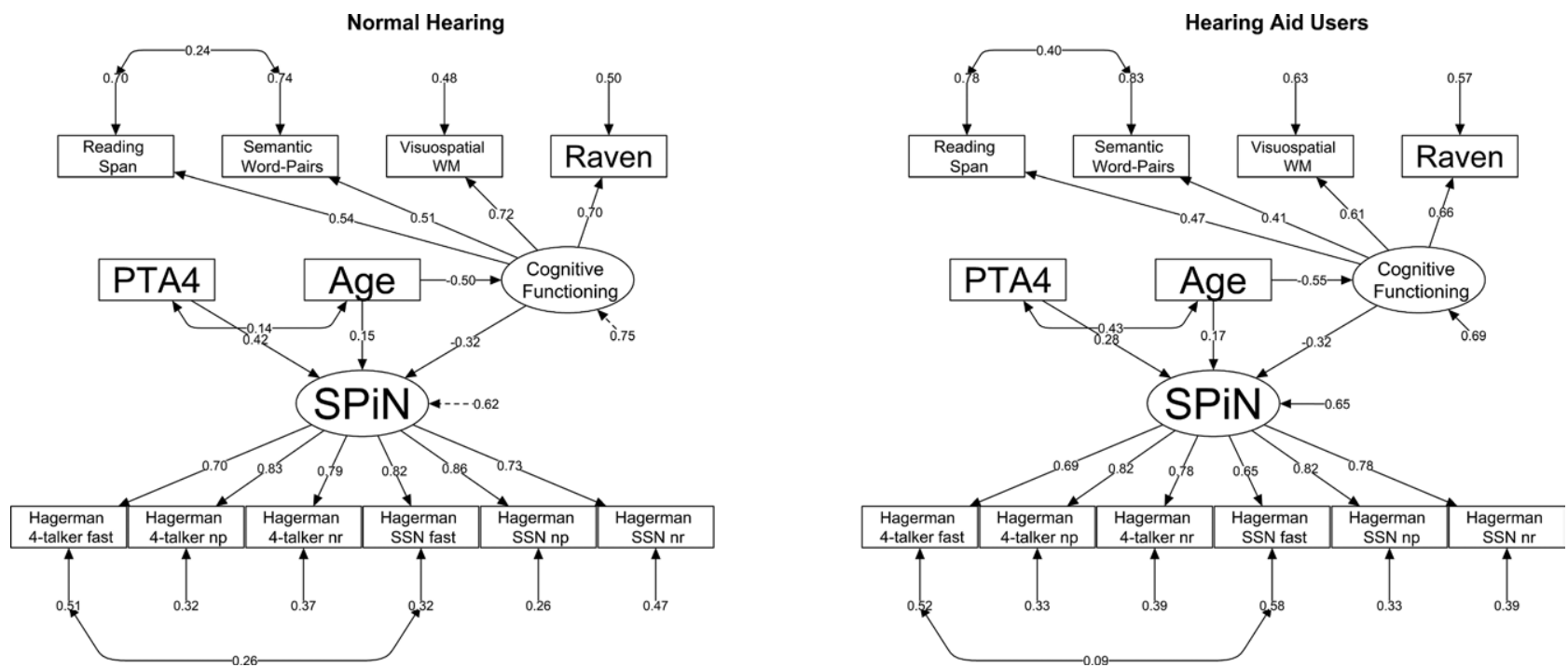

Fig. 3. Structural equation model for both groups. Note that the figure is showing the standardized coefficients although the final model, shown here, has the unstandardized coefficients constrained to be equal across the two groups.

TABLE 3. Fit statistics for each model, with the final model at the last line

\begin{tabular}{lccccrrr}
\hline Model & $\chi^{2}$ & $d f$ & RMSEA (95\% Cl) & TLI & \multicolumn{1}{c}{ CFI } & BIC \\
\hline Model 1 & 197.59 & 102 & $0.07(0.05-0.08)$ & 0.94 & 0.95 & 22806.33 \\
Model 2 & 160.41 & 100 & $0.06(0.04-0.07)$ & 0.96 & 0.97 & 22781.13 & $<0.001$ \\
Model 3 & 149.88 & 98 & $0.05(0.03-0.07)$ & 0.96 & 0.97 & 22782.57 & 0.001 \\
Model 4 & 156.4 & 106 & $0.05(0.03-0.06)$ & 0.97 & 0.97 & 22741.18 \\
Model 5 & 150.09 & 102 & $0.05(0.03-0.06)$ & 0.97 & 0.97 & 22758.84 & 0.001 \\
Final model & 158.91 & 110 & $0.05(0.03-0.06)$ & 0.97 & 0.97 & 22719.74 \\
\hline
\end{tabular}

Note. In the first model, Model 1, all parameters are set free between the groups. The second model, Model 2, is the same as Model 1 with the addition of covariance between the error terms of Semantic Word-Pairs and Reading Span. Model 3 is built on Model 1 and Model 2 with the addition of covariance of the error terms for Hagerman fast, 4 -talker and SSN. Model 4 and 5 are constrained to have equal factor loadings and regression weights, respectively, across the groups. The final model is built on model 3 with additional constraints both the factor loadings and regression weights.

BIC, Bayesian Information Criterion; CFI, comparative fit index; RMSEA, root mean square error of approximation; TLI, Tucker-Lewis index.

speech-in-noise performance in unaided listening conditions. The present study's findings are in line with previous research showing that a range of cognitive functions is related to performance in speech-in-noise tests. A majority of the research has examined the effects of cognition in groups of people with hearing loss (e.g., Arehart et al. 2013; Foo et al. 2007; Lunner 2003). This relationship has also been found in young normal-hearing persons in some studies (e.g., Michalek et al. 2018; Stenbäck et al. 2015, 2021) and older normal-hearing (Füllgrabe et al. 2015). Here, we extend this research by using statistical techniques that enable us to model this and compare

TABLE 4. Results from the LRTs

\begin{tabular}{lcrrr}
\hline & Models & & & \\
Type of Test & Compared & LRT & \multicolumn{1}{c}{$p$} & $d f^{2}$ \\
\hline Model optimization & 2 vs. 1 & 37.17 & $<0.01$ & 2 \\
Invariance testing & 3 vs. 2 & 10.53 & $<0.01$ & 2 \\
& 4 vs. 3 & 0.22 & 0.99 & 4 \\
& 5 vs. 4 & 6.52 & 0.59 & 8 \\
& 6 vs. 5 & 8.82 & 0.36 & 8
\end{tabular}

Note. Model 2 and 3, are optimized after modification indices and model 4 to 6 have the factor loadings, regression weights, and both factor loadings and regression weights constrained. LRT, likelihood ratio test. the effects between groups (i.e., normal-hearing and hearingimpaired older individuals).

In light of the recent meta-analyses, the relationship between the latent constructs cognitive functioning and speech-in-noise in our study is, at least, similar to the results of Dryden et al. (2017). In their meta-analysis, the correlation was around 0.2 for most cognitive measures. Note that Dryden et al. (2017) included studies focusing on unaided hearing in both participants with and without hearing loss which might make it difficult to interpret their results. However, in the meta-analysis by Füllgrabe and Rosen (2016b), the aggregated correlation across the included studies was 0.12 . In our study, the Beta weight between cognitive functioning and SPiN was of small to medium strength. This is in line with the strength of the findings in the study by Dryden et al. (2017) but not the study of Füllgrabe and Rosen (2016b). It is worth pointing out here that in the latter study, the results were true only in the younger normal-hearing adults, and in the current study, the mean age was around 61 in both groups. However, considering the Dryden et al. (2017) meta-analysis, in which all age groups, as well as hearing status, remained significant, one could speculate that these findings generalize to younger individuals as well. Worth noting is that the speech-in-noise test used in the current study 
TABLE 5. Standardized regression coefficients and confidence intervals for both groups

\begin{tabular}{lllc}
\hline Group & Latent Variable & Regressor & $\beta(95 \%$ Cl) \\
\hline Normal Hearing & SPiN & Cognitive Functioning & $-0.32(-0.46$ to -0.18$)$ \\
& SPiN & PTA4 & $0.42(0.25$ to 0.59$)$ \\
& SPiN & Age & $0.15(-0.02$ to 0.32$)$ \\
Hearing Aid Users & Cognitive Functioning & Age & $-0.5(-0.62$ to -0.38$)$ \\
& SPiN & Cognitive Functioning & $-0.32(-0.46$ to -0.18$)$ \\
& SPiN & PTA4 & $0.28(0.15$ to 0.4$)$ \\
& SPiN & Age & $0.17(-0.02$ to 0.35$)$ \\
& Cognitive Functioning & Age & $-0.55(-0.69$ to -0.42$)$ \\
\hline
\end{tabular}

may not generalize very well to everyday communication due to the low semantic context and stylized structure. However, the current study's findings indicate that the ability to recognize speech in background noise successfully is better in people with higher cognitive capacity.

The findings are also in line with a plethora of research showing the effects of aging on speech-in-noise performance and auditory functions like hearing thresholds. For example, it has been found that aging has an impact on hearing thresholds (e.g., Gates \& Cooper 1991; Wiley et al. 2008), as well as auditory temporal processing (Fitzgibbons \& Gordon-Salant 2001; Pichora-Fuller et al. 2006). Finally, in a recent study by Danielsson et al. (2019), it was found that age affected auditory tasks such as temporal-order identification. Moreover, the results from the present study are also in line with research showing that age affects cognitive functioning. For example, using factor analysis, Humes et al. (2013) found that age has a negative effect on cognitive functioning (i.e., Reading span, Visuospatial WM, Semantic-Word Pairs, and Raven's). However, this effect was not evident when sensory processing was used as a control.

The final model of the present research shows that aging effects on cognitive functions and speech identification are almost similar across normal-hearing and hearing-impaired older groups. We reason that this pattern of results is in line with the common-cause model of aging (e.g., Lindenberger \& Baltes 1994), assuming a domain-general neurobiological mechanism affecting sensorimotor functioning (like speech identification in degraded listening conditions). The main result of the current study supports the notion that cognitive functioning is important for listening in adverse conditions, regardless of hearing status. Importantly, we found that cognitive functioning is crucial at $50 \%$ word recognition, whereas a lot of research has shown effects at $80 \%$ word recognition, or higher, SRT (e.g., Larsby et al. 2011; Stenbäck et al. 2015, 2016). That cognitive processes are crucial for adverse listening conditions is further in line with the ELU model (e.g., Rönnberg et al. 2013, 2019). According to the ELU, a mismatch between the input and the long-term representations, the RAMBPHO buffer feeds it forward to WM and executive functions (Rönnberg et al. 2013, 2019). Furthermore, all tasks used in the current study (especially the WM test) are dual tasks. According to the ELU model, understanding speech involves both a storage and a processing component. Specifically, according to the ELU model, the storage contains semantic and/or sensory information, and the processing component deals with grammatical information. Our results further support this notion.

However, it should be noted that when we investigate the effects of hearing loss on different kinds of memory systems such as working memory, episodic and semantic long-term memory, there is a selectivity in the negative effects of hearing loss that cannot be unexplained by age-confounding (Rönnberg et al. 2011, 2014, 2021). So, in essence, the common cause account does not receive unequivocal support from these data sets. From the point of the ELU model, this is explained by the notion of relative disuse/use of the memory systems by people with hearing loss in the process of speech intelligibility in noise (see Rönnberg et al. 2021 for an in-depth explanation). Thus, we end up with a theoretical model that may, in general, be relatively independent of hearing loss for the general importance of cognition on speech in noise intelligibility, but that different memory systems and how they interact may be differentially sensitive to hearing loss in their contribution to speech communication and understanding.

\section{CONCLUSION}

In this study, we modeled the effect of age and cognitive functioning on speech-in-noise performance using a multigroup approach. Cognitive functioning was measured by a range of working memory tasks as well as fluid intelligence, which is psychological constructs all thought to be highly related. Speechin-noise, in turn, was measured by signal-to-noise ratios in the Hagerman test ( $50 \%$ word recognition). At the beginning of the modeling phase, the models were set up with entirely free parameters between two groups (hearing impaired and normal hearing). We then continued and developed the model to serve our aim: to examine whether there are different effects of cognitive functioning on speech-in-noise performance. To this end, we restrained the regression weights between the latent variables and found that this improved the fit of the model. Our findings revealed similar cognitive functions and aging effects on speech-in-noise performance in normal-hearing and aided hearing-impaired groups.

\section{ACKNOWLEDGMENTS}

The research was supported by three grants: the Linnaeus Centre HEAD excellence center grant (349-2007-8654) from the Swedish Research Council, a program grant from FORTE (2012-1693), awarded to JR as PI, and a grant from the Swedish Research Council, awarded to Dr. Fridberger (VR-2017-06092). All authors contributed equally to this work. JR designed the project, and the main conceptual ideas (i.e., the n200 study). All authors contributed to the formalization of the model tested in the manuscript. EM prepared the data, carried out the data analysis, and wrote the original draft (except for the Methods section, which VS wrote). JR and HD supervised the project. HD assisted with the data analysis. JR, HD, VS, and SM authors contributed to the review and editing of the manuscript.

The authors have no conflicts of interest relevant to this article to disclose. 
Address for correspondence: Erik Marsja, Department of Behavioural Sciences and Learning, Linköping University, S-581 83, Linköping, Sweden. E-mail: erik.marsja@liu.se

Received March 31, 2021; accepted November 27, 2021

\section{REFERENCES}

Ackerman, P. L., Beier, M. E., Boyle, M. O. (2002). Individual differences in working memory within a nomological network of cognitive and perceptual speed abilities. J Exp Psychol Gen, 131, 567-589.

Akeroyd, M. A. (2008). Are individual differences in speech reception related to individual differences in cognitive ability? A survey of twenty experimental studies with normal and hearing-impaired adults. Int $J$ Audiol, 47(Sup2), S53-S71.

Allén, S. (1970). Frequency Dictionary of Present-Day Swedish (In Swedish: Nusvensk Frekvensbok). Almquist and Wiksell.

Arehart, K. H., Souza, P., Baca, R., Kates, J. M. (2013). Working memory, age, and hearing loss: susceptibility to hearing aid distortion. Ear Hear, 34, 251-260.

Aust, F., \& Barth, M. (2020). Papaja: Prepare Reproducible Apa Journal Articles With R Markdown. R package version 0.1.0.9997. https://github. $\mathrm{com} / \mathrm{crsh} /$ papaja

Baddeley, A. D. (2012). Working memory: theories, models, and controversies. Annu Rev Psychol, 63, 1-29.

Baltes, P. B., \& Lindenberger, U. (1997). Emergence of a powerful connection between sensory and cognitive functions across the adult life span: a new window to the study of cognitive aging? Psychol Aging, 12, $12-21$.

Besser, J., Festen, J. M., Goverts, S. T., Kramer, S. E., Pichora-Fuller, M. K. (2015). Speech-in-speech listening on the LiSN-S test by older adults with good audiograms depends on cognition and hearing acuity at high frequencies. Ear Hear, 36, 24-41.

Brand, T. (2000). Analysis and Optimization of Psychophysical Procedures in Audiology. BIS Verlag.

Cardin, V. (2016). Effects of aging and adult-onset hearing loss on cortical auditory regions. Front Neurosci, 10, 199.

Conway, A. R. A., Cowan, N., Bunting, M. F., Therriault, D. J., Minkoff, S. R. B. (2002). A latent variable analysis of working memory capacity, short-term memory capacity, processing speed, and general fluid intelligence. Intelligence, 30, 163-183.

Croll, P. H., Vinke, E. J., Armstrong, M. N., Licher, S., Vernooij, M. W., et al. (2021). Hearing loss and cognitive decline in the general population: a prospective cohort study. $J$ Neurol, 26, 860-871.

Danielsson, H., Humes, L. E., Rönnberg, J. (2019). Different associations between auditory function and cognition depending on type of auditory function and type of cognition. Ear Hear, 40, 1210-1219.

Dimitrijevic, A., John, M. S., Picton, T. W. (2004). Auditory steady-state responses and word recognition scores in normal-hearing and hearingimpaired adults. Ear Hear, 25, 68-84.

Dryden, A., Allen, H. A., Henshaw, H., Heinrich, A. (2017). The association between cognitive performance and speech-in-noise perception for adult listeners: a systematic literature review and meta-analysis. Trends Hear, $21,2331216517744675$.

Enders, C., \& Bandalos, D. (2001). The relative performance of full information maximum likelihood estimation for missing data in structural equation models. Struct Equ Modeling, 8, 430-457.

Engle, R. W., Tuholski, S. W., Laughlin, J. E., Conway, A. R. A. (1999). Working memory, short-term memory, and general fluid intelligence: a latent-variable approach. J Exp Psychol Gen, 128, 309-331.

Epskamp, S. (2019). semPlot: Path Diagrams and Visual Analysis of Various SEM Packages' Output. R package version 1.1.2. https://CRAN.Rproject.org/package $=$ semPlot

Fitzgibbons, P. J., \& Gordon-Salant, S. (2001). Aging and temporal discrimination in auditory sequences. J Acoust Soc Am, 109, 2955-2963.

Folstein, M. F., Folstein, S. E., McHugh, P. R. (1975). 'Mini-mental state". A practical method for grading the cognitive state of patients for the clinician. J Psychiatr Res, 12, 189-198.

Foo, C., Rudner, M., Rönnberg, J., Lunner, T. (2007). Recognition of speech in noise with new hearing instrument compression release settings requires explicit cognitive storage and processing capacity. J Am Acad Audiol, 18, 618-631.

Füllgrabe, C., Moore, B. C. J., Stone, M. A. (2015). Age-group differences in speech identification despite matched audiometrically normal hearing: contributions from auditory temporal processing and cognition. Front Aging Neurosci, 7, 1-25.

Füllgrabe, C., \& Rosen, S. (2016a). Investigating the Role of Working Memory in Speech-in-noise Identification for Listeners with Normal Hearing. In P. van Dijk, D. Başkent, E. Gaudrain, E. de Kleine, A. Wagner, \& C. Lanting (Eds.), Physiology, Psychoacoustics and Cognition in Normal and Impaired Hearing (pp. 29-36). Springer International Publishing.

Füllgrabe, C., \& Rosen, S. (2016b). On the (un)importance of working memory in speech-in-noise processing for listeners with normal hearing thresholds. Front Psychol, 7, 1268.

Gates, G. A., \& Cooper, J. C. (1991). Incidence of hearing decline in the elderly. Acta Otolaryngol, 111, 240-248.

Gordon-Salant, S., \& Cole, S. S. (2016). Effects of age and working memory capacity on speech recognition performance in noise among listeners with normal hearing. Ear Hear, 37, 593-602.

Graham, E. R., \& Burke, D. M. (2011). Aging increases inattentional blindness to the gorilla in our midst. Psychol Aging, 26, 162-166.

Hagerman, B. (1982). Sentences for testing speech intelligibility in noise. Scand Audiol, 11, 79-87.

Hagerman, B., \& Kinnefors, C. (1995). Efficient adaptive methods for measuring speech reception threshold in quiet and in noise. Scand Audiol, 24, 71-77.

Hu, L. T., \& Bentler, P. M. (1999). Cutoff criteria for fit indexes in covariance structure analysis: Conventional criteria versus new alternatives. Struct Equ Modeling, 6, 1-55.

Humes, L. E., Watson, B. U., Christensen, L. A., Cokely, C. G., Halling, D. C., Lee, L. (1994). Factors associated with individual differences in clinical measures of speech recognition among the elderly. J Speech Hear Res, 37, 465-474.

Humes, L. E. (2002). Factors underlying the speech-recognition performance of elderly hearing-aid wearers. J Acoust Soc Am, 112(3 Pt 1), $1112-1132$.

Humes, L. E., Busey, T. A., Craig, J., Kewley-Port, D. (2013). Are agerelated changes in cognitive function driven by age-related changes $\mathrm{i}$ sensory processing? Atten Percept Psychophys, 75, 508-524.

Husain, F. T., Medina, R. E., Davis, C. W., Szymko-Bennett, Y., Simonyan, K., Pajor, N. M., Horwitz, B. (2011). Neuroanatomical changes due to hearing loss and chronic tinnitus: a combined VBM and DTI study. Brain Res, 1369, 74-88.

Jerger, J., Jerger, S., Oliver, T., Pirozzolo, F. (1989). Speech understanding in the elderly. Ear Hear, 10, 79-89.

Kane, M. J., Hambrick, D. Z., Tuholski, S. W., Wilhelm, O., Payne, T. W., Engle, R. W. (2004). The generality of working memory capacity: a latent-variable approach to verbal and visuospatial memory span and reasoning. J Exp Psychol Gen, 133, 189-217.

Kyllonen, P. C., \& Christal, R. E. (1990). Reasoning ability is (little more than) working-memory capacity?! Intelligence, 14, 389-433.

Larsby, B., Hällgren, M., Lyxell, B. (2008). The interference of different background noises on speech processing in elderly hearing impaired subjects. Int J Audiol, 47(Suppl. 2), 83-90.

Larsby, B., Hällgren, M., Lyxell, B. (2011). The role of working memory capacity and speed of lexical access in speech recognition in noise. Proceedings of ISAAR 2011, 30, 234-237.

Lindenberger, U., \& Baltes, P. B. (1994). Sensory functioning and intelligence in old age: a strong connection. Psychol Aging, 9, 339-355.

Lin, L. C., Huang, P. H., Weng, L. J. (2017). Selecting Path Models in SEM: A Comparison of Model Selection Criteria. Struct Equ Modeling, 24, 855-869.

Lunner, T. (2003). Cognitive function in relation to hearing aid use. Int $J$ Audiol, 42 (Suppl 1), S49-S58.

Lunner, T., \& Sundewall-Thorén, E. (2007). Interactions between cognition, compression, and listening conditions: effects on speech-in-noise performance in a two-channel hearing aid. J Am Acad Audiol, 18, 604-617.

MacCallum, R. C., Browne, M. W., Sugawara, H. M. (1996). Power analysis and determination of sample size for covariance structure modeling. Psychol Methods, 1, 130-149.

McAvinue, L. P., Habekost, T., Johnson, K. A., Kyllingsbæk, S., Vangkilde, S., Bundesen, C., Robertson, I. H. (2012). Sustained attention, attentional selectivity, and attentional capacity across the lifespan. Atten Percept Psychophys, 74, 1570-1582.

Meister, H., Schreitmüller, S., Grugel, L., Ortmann, M., Beutner, D., Walger, M., Meister, I. G. (2013). Cognitive resources related to speech recognition with a competing talker in young and older listeners. Neuroscience, $232,74-82$. 
Michalek, A. M. P., Ash, I., Schwartz, K. (2018). The independence of working memory capacity and audiovisual cues when listening in noise. Scand J Psychol, 59, 578-585.

Moradi, S., Lidestam, B., Hällgren, M., Rönnberg, J. (2014). Gated auditory speech perception in elderly hearing aid users and elderly normal-hearing individuals: effects of hearing impairment and cognitive capacity. Trends Hear, 18, 2331216514545406.

Moore, D. R., Edmondson-Jones, M., Dawes, P., Fortnum, H., McCormack, A., Pierzycki, R. H., Munro, K. J. (2014). Relation between speech-innoise threshold, hearing loss and cognition from 40-69 years of age. PLoS One, 9, e107720.

Peelle, J. E., Troiani, V., Grossman, M., Wingfield, A. (2011). Hearing loss in older adults affects neural systems supporting speech comprehension. J Neurosci, 31, 12638-12643.

Pichora-Fuller, M. K., Schneider, B. A., Benson, N. J., Hamstra, S. J., Storzer, E. (2006). Effect of age on detection of gaps in speech and nonspeech markers varying in duration and spectral symmetry. J Acoust Soc Am, 119, 1143-1155

Putnick, D. L., \& Bornstein, M. H. (2016). Measurement invariance conventions and reporting: The state of the art and future directions for psychological research. Dev Rev, 41, 71-90.

Pronk, M., Lissenberg-Witte, B. I., van der Aa, H. P. A., Comijs, H. C., Smits, C., Lemke, U., Zekveld, A. A., Kramer, S. E. (2019). Longitudinal relationships between decline in speech-in-noise recognition ability and cognitive functioning: the longitudinal aging study amsterdam. $J$ Speech Lang Hear Res, 62(4S), 1167-1187.

Raven, J. (2000). The Raven's progressive matrices: change and stability over culture and time. Cogn Psychol, 41, 1-48.

R Core Team. (2021). R: A Language and Environment for Statistical Computing. R Foundation for Statistical Computing. https://www.r-project.org/

Rosseel, Y. (2012). lavaan: An R package for structural equation modeling. J Stat Softw, 48, 1-36. http://www.jstatsoft.org/v48/i02/paper

Rönnberg, J., Arlinger, S., Lyxell, B., Kinnefors, C. (1989). Visual evoked potentials: relation to adult speechreading and cognitive function. $J$ Speech Hear Res, 32, 725-735.

Rönnberg, J., Holmer, E., Rudner, M. (2019). Cognitive hearing science and ease of language understanding. Int J Audiol, 58, 247-261.

Rönnberg, J., Holmer, E., Rudner, M. (2021). Cognitive hearing science: three memory systems, two approaches, and the ease of language understanding model. J Speech Lang Hear Res, 64, 359-370.

Rönnberg, J., Lunner, T., Ng, E. H., Lidestam, B., Zekveld, A. A., Sörqvist, P., Lyxell, B., Träff, U., Yumba, W., Classon, E., Hällgren, M., Larsby,
B., Signoret, C., Pichora-Fuller, M. K., Rudner, M., Danielsson, H., Stenfelt, S. (2016). Hearing impairment, cognition and speech understanding: exploratory factor analyses of a comprehensive test battery for a group of hearing aid users, the n200 study. Int J Audiol, 55, 623-642.

Rönnberg, J., Lunner, T., Zekveld, A., Sörqvist, P., Danielsson, H., Lyxell, B., Dahlström, Ö., Signoret, C., Stenfelt, S., Pichora-Fuller, M. K., Rudner, M. (2013). The Ease of Language Understanding (ELU) model: theoretical, empirical, and clinical advances. Front Syst Neurosci, 7, 1-17.

Salthouse, T. A., \& Babcock, R. L. (1991). Decomposing adult age differences in working memory. Dev Psychol, 27, 763-776.

Satorra, A., \& Bentler, P. M. (2001). A scaled difference chi-square test statistic for moment structure analysis. Psychometrika, 66, 507-514.

Schafer, J. L., \& Graham, J. W. (2002). Missing data: our view of the state of the art. Psychol Methods, 7, 147-177.

Schoof, T., \& Rosen, S. (2014). The role of auditory and cognitive factors in understanding speech in noise by normal-hearing older listeners. Front Aging Neurosci, 6, 307.

Steiger, J. H. (2007). Understanding the limitations of global fit assessment in structural equation modeling. Pers Individ Dif, 42, 893-898.

Stenbäck, V., Marsja, E., Hällgren, M., Lyxell, B., Larsby, B. (2021). The contribution of age, working memory capacity, and inhibitory control on speech recognition in noise in young and older adult listeners. $J$ Speech Lang Hear Res, 64, 4513-4523.

Stenbäck, V., Hällgren, M., Larsby, B. (2016). Executive functions and working memory capacity in speech communication under adverse conditions. Speech Lang Hear, 19, 218-226.

Stenbäck, V., Hällgren, M., Lyxell, B., Larsby, B. (2015). The Swedish Hayling task, and its relation to working memory, verbal ability, and speech-recognition-in-noise. Scand J Psychol, 56, 264-272.

Unsworth, N., \& Engle, R. W. (2005). Working memory capacity and fluid abilities: examining the correlation between operation span and raven. Intelligence, 33, 67-81.

Vrieze, S. I. (2012). Model selection and psychological theory: a discussion of the differences between the Akaike information criterion (AIC) and the Bayesian information criterion (BIC). Psychol Methods, 17, 228-243.

Wiley, T. L., Chappell, R., Carmichael, L., Nondahl, D. M., Cruickshanks, K. J. (2008). Changes in hearing thresholds over 10 years in older adults. J Am Acad Audiol, 19, 281-292; quiz 371.

Wingfield, A., Stine, E. A., Lahar, C. J., Aberdeen, J. S. (1988). Does the capacity of working memory change with age? Exp Aging Res, 14, 103-107. 